\title{
Star Unfolding from a Geodesic Curve
}

\author{
Stephen Kiazyk and Anna Lubiw \\ Cheriton School of Computer Science \\ University of Waterloo, Waterloo, ON, Canada \\ \{skiazk, alubiw\}@uwaterloo.ca
}

\begin{abstract}
There are two known ways to unfold a convex polyhedron without overlap: the star unfolding and the source unfolding, both of which use shortest paths from vertices to a source point on the surface of the polyhedron. Non-overlap of the source unfolding is straightforward; non-overlap of the star unfolding was proved by Aronov and O'Rourke in 1992. Our first contribution is a much simpler proof of non-overlap of the star unfolding.

Both the source and star unfolding can be generalized to use a simple geodesic curve instead of a source point. The star unfolding from a geodesic curve cuts the geodesic curve and a shortest path from each vertex to the geodesic curve. Demaine and Lubiw conjectured that the star unfolding from a geodesic curve does not overlap. We prove a special case of the conjecture. Our special case includes the previously known case of unfolding from a geodesic loop. For the general case we prove that the star unfolding from a geodesic curve can be separated into at most two non-overlapping pieces.
\end{abstract}

1998 ACM Subject Classification I.3.5 Computational Geometry and Object Modeling

Keywords and phrases unfolding, convex polyhedra, geodesic curve

Digital Object Identifier 10.4230/LIPIcs.SOCG.2015.390

\section{Introduction}

An unfolding of a polyhedron $\mathcal{P}$ is obtained by cutting the surface of $\mathcal{P}$ in such a way that it can be flattened into the plane, forming a single polygon. A main goal is to find unfoldings that are simple, that is, do not self-overlap. If we have an unfolding that does not overlap, we can make a model of the polyhedron from a sheet of paper by cutting the outline of the polygon and gluing appropriate pairs of edges together.

Unfoldings have fascinated people since the time of Dürer's beautiful examples [6]. A long-standing open question is whether every convex polyhedron has a non-overlapping edge unfolding, where only edges of the polyhedron are cut. However, if we allow cuts that cross faces - which is the model used in this paper - then there are several known methods to unfold convex polyhedra without overlap.

Unfoldings of polyhedra have applications in product manufacturing, for constructing a 3-dimensional object from a sheet of metal or plastic, and also in graphics for applying texture mapping, where 2-dimensional image coordinates must be assigned to points on a 3-dimensional model. Unfolding is also applied as a theoretical tool for the study of shortest paths on the surface of a polyhedron.

There are two main methods known to unfold convex polyhedra without overlap, both defined in terms of shortest paths on the polyhedron surface to a "source" point $x$. A fundamental property of shortest paths on the surface of a convex polyhedron is that they unfold to straight-line segments. More generally, any geodesic (or locally shortest) path on the surface of a polyhedron unfolds to a straight-line segment.

c) (i) $\odot$ Stephen Kiazyk and Anna Lubiw;

licensed under Creative Commons License CC-BY

31st International Symposium on Computational Geometry (SoCG'15).

Editors: Lars Arge and János Pach; pp. 390-404

Leibniz International Proceedings in Informatics

LI PICS Schloss Dagstuhl - Leibniz-Zentrum für Informatik, Dagstuhl Publishing, Germany 


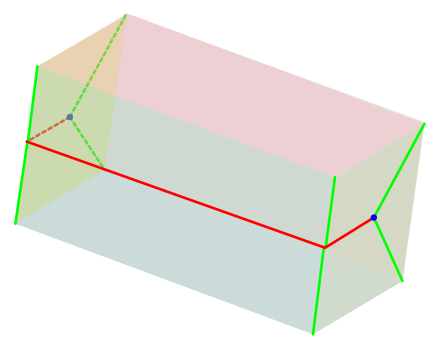

(a)

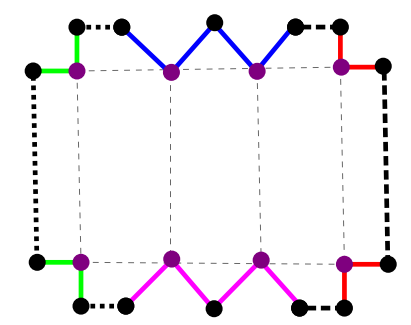

(b)

Figure 1 An example star unfolding from a geodesic curve (in red) on a rectangular box. Faint dashed lines inside the unfolding indicate some of the original edges.

The star unfolding is obtained by cutting a shortest path from every vertex of the polyhedron to the point $x$. The cuts form a star at $x$, hence the name. The source unfolding is obtained by cutting the ridge tree (also known as the cut locus), the locus of points that have more than one shortest path to $x$. It is easy to see that the source unfolding does not overlap, because all the shortest paths from $x$ to points on the surface unfold into straight lines radiating from $x$. The star and source unfoldings are dual in the sense that the pieces of the surface delimited by the ridge tree and the vertex-to-source shortest path cuts are joined at "opposite ends": in the star unfolding the pieces are joined at the ridge tree; and in the source unfolding the pieces are joined at the source point $x$.

Alexandrov thought the star unfolding might overlap (see [5]); the surprising result that it does not was proved by Aronov and O'Rourke [2]. Their proof is by induction and involves combining two vertices into one in a kind of "Alexandrov surgery." The proof is long, and Demaine and O'Rourke, in their book, "Geometric Folding Algorithms" [5] call the proof "not straightforward" and omit it.

Our first main result is a new proof of non-overlap of the star unfolding that is more straightforward. We do not modify the polyhedron or appeal to Alexandrov's gluing theorem.

The star and source unfoldings can be generalized in a natural way by using a simple geodesic curve $\lambda$ instead of a source point $x$. For the source unfolding, we cut the ridge tree (the locus of points that have more than one shortest path to the curve $\lambda$ ), and for the star unfolding we cut the curve $\lambda$ itself and a shortest path from every vertex of the polyhedron to $\lambda$. See Figure 1. Such generalizations were first introduced by Itoh et al. [9] who proved non-overlap results for the case of closed curves (see also $[11,8]$ ). Demaine and Lubiw [4, Lemma 1] proved non-overlap of the source unfolding for (open) geodesic curves, and conjectured the same for the star unfolding.

Our second main result is a special case of this conjecture: we prove that the star unfolding from a geodesic curve unfolds without overlap if the curve is "balanced" (as defined in Section 3). The balance condition automatically holds for the point star unfolding.

We give two implications of our second result. The first is that the star unfolding does not overlap if the curve is a geodesic loop, meaning the curve endpoints $a$ and $b$ are (almost) coincident. In the limit when $a=b$ the unfolding consists of two pieces joined at a point. This gives an alternative to the result of Itoh et al. [9] that the star unfoldings of the inside and the outside of a geodesic loop do not overlap, and that the two pieces may be joined into one non-overlapping piece. Their proof that the outside unfolds without overlap had a flaw; our result repairs it. We can extend this result (and our other results) from geodesics to quasigeodesics, to be defined below.

The second consequence of our result is that every star unfolding from a geodesic curve 


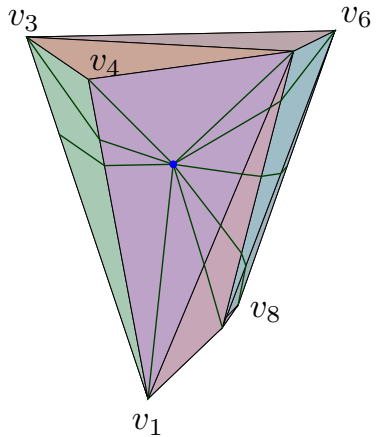

(a)

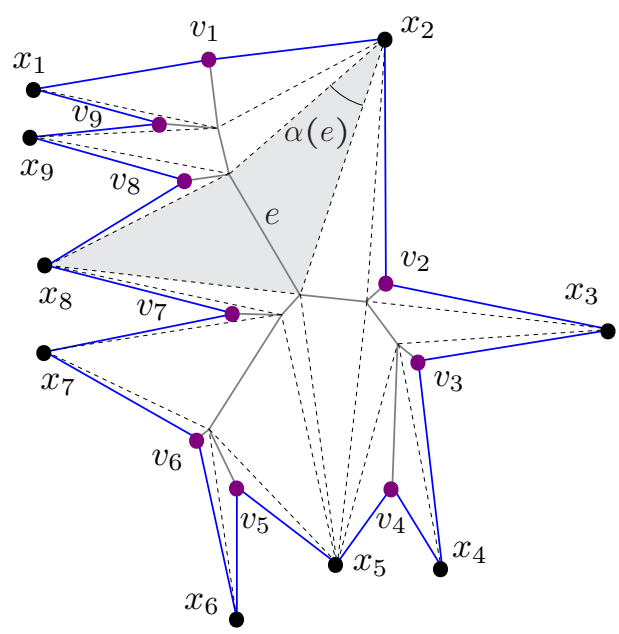

(b)

Figure 2 The star unfolding from a point. (a) The polyhedron, the source point, and the shortest paths from vertices to the source point. (b) The corresponding star unfolding. The ridge tree is shown in grey. The kites are shown with dashed lines. The kite on ridge tree edge $e$ is shaded, and its source angle $\alpha(e)$ is indicated. Note that the unfolding shows the inside surface of the polyhedron.

can be cut into two pieces such that each piece is non-overlapping. The extra cuts consist of shortest paths from a point on the ridge tree to the curve.

To conclude this section we mention a few reasons to explore new unfoldings of convex polyhedra. One is to find "nicer" unfoldings. As the number of vertices of a polyhedron increases, the star unfolding from a point becomes very spiky with many sharp angles, for example see [1, Figure 7]. By contrast, in the star unfolding from a geodesic curve many (or in some cases, all) vertices may have shortest paths to interior points of the curve, resulting in many $90^{\circ}$ angles and fewer sharp angles. New unfolding methods for convex polyhedra might also shed light on the case of non-convex polyhedra. Having a larger repertoire of unfoldings also opens the door to optimization, e.g., minimize the area of a bounding box of the unfolding, or minimize the total cut length, or maximize the minimum angle.

Geodesic star unfoldings may also have implications for the conjecture [3] that every convex polyhedron has a general zipper unfolding, a non-overlapping unfolding formed by cutting a single path on the polyhedron surface. If quasigeodesic star unfoldings do not overlap, then it would suffice to find a quasigeodesic curve that goes through all the vertices.

\subsection{Preliminaries and Definitions}

- Definition 1. Let $\mathcal{P}$ be a convex polyhedron, and let $x$ be a point on $\mathcal{P}$. The star unfolding, $S_{x}$, is a 2 -dimensional polygon formed by cutting $\mathcal{P}$ along a shortest path from every vertex of $\mathcal{P}$ to $x$, and flattening the result into the plane. See Figure 2. Note that there is a choice of cuts if a vertex has more than one shortest path to $x$.

If $\mathcal{P}$ has $n$ vertices, then the polygon $S_{x}$ will have $2 n$ vertices in general, and $2(n-1)$ vertices if $x$ is located at a vertex of $\mathcal{P}$. The vertices of $S_{x}$ alternate around the boundary between vertex images, denoted $v_{i}$, that correspond to the vertices of $\mathcal{P}$, and $n$ 'copies' of $x$, called source images and denoted by $x_{i}$. See Figure 2. 
The edges of $S_{x}$ correspond to the shortest path cuts made from each vertex to $x$. Therefore, the two edges incident to any vertex image $v_{i}$ are always the same length.

The ridge tree (or "cut locus"), $T_{x}$, is the closure of the set of all points on the surface of $\mathcal{P}$ that have more than one shortest path to $x$. It is known that the ridge tree is in fact a tree [12], and that its edges are shortest paths [1] and thus correspond to straight-line segments in $S_{x}$. See Figure 2(b). As a corollary to their proof of non-overlap [2, Theorem 10.2], Aronov and O'Rourke proved that the ridge tree is a subset of the Voronoi diagram of the images of $x$.

Readers interested only in the point case may proceed directly to Section 2 . In the remainder of this section we give definitions for the star unfolding from a geodesic curve.

Given a polyhedron $\mathcal{P}$, a geodesic curve $\lambda$ on $\mathcal{P}$ is a curve on the surface of $\mathcal{P}$, such that at every interior point $p$ of $\lambda$, the surface angle to either side of $p$ is exactly $\pi$. If the surface angle to either side of $p$ is at most $\pi$ then the curve is called a quasigeodesic. We will only consider [quasi]geodesic curves that are simple, meaning there is no point of self-intersection between any two interior (i.e., non-endpoint) points of the curve.

- Definition 2. Let $\mathcal{P}$ be a convex polyhedron, and let $\lambda$ be a simple geodesic curve on the surface of $\mathcal{P}$. The geodesic star unfolding $S_{\lambda}$ is a 2-dimensional polygon formed by cutting $\lambda$, and cutting a shortest path along the surface of $\mathcal{P}$ from every vertex $v_{i}$ of $\mathcal{P}$ to $\lambda$, and flattening the result into the plane. See Figure 5.

The endpoints of $\lambda$ are labelled $a$ and $b$ and the two sides of $\lambda$ are labelled $s$ and $t$, with the convention that the clockwise order around $\lambda$ on the outside surface of $\mathcal{P}$ is $a, s, b, t$. We distinguish shortest paths to $\lambda$ that arrive at (or "report to") $a$ or $b$, and shortest paths that arrive at interior points of $\lambda$ on side $s$ or $t$. Any shortest path that arrives at an interior point of $\lambda$ forms a right angle with $\lambda$, as shown by the following lemma, adapted from Ieiri et al. [7, Corollary 1].

- Lemma 3. Let $\lambda$ be a geodesic curve on a convex polyhedron $\mathcal{P}$, with points $x_{0}$ on $\lambda$ and $x$ not on $\lambda$ such that $x x_{0}$ is a shortest path from $x$ to $\lambda$. The angles formed between $x x_{0}$ and $\lambda$ are at least $\frac{\pi}{2}$ to each side.

In the unfolding $S_{\lambda}$, a source image is either a copy of an endpoint ( $a$ or $b$ ), called a point image, or a sub-segment of $\lambda$ corresponding to side $s$ or $t$, called a segment image. (Note that a segment image might include one of the endpoints of $\lambda$.) Around a clockwise traversal of the boundary of $S_{\lambda}$ we encounter source images in order $a, s, b, t$ - this is because our convention is to draw the inside of the polyhedron's surface facing up. Each consecutive pair of source images is separated by two edges (of equal length) joined at a vertex image, such that the edges correspond to the shortest path cut at the vertex.

The ridge tree of a geodesic $\lambda$, denoted $T_{\lambda}$, is the closure of the set of all points on the surface of $\mathcal{P}$ that have more than one shortest path to $\lambda$. That the ridge tree is an actual tree was established by Demaine and Lubiw [4, Lemma 4]. (In fact, their lemma applies to the outside of any closed convex curve on the surface of $\mathcal{P}$-in the present situation, the closed convex curve is the set of points at some fixed distance $\epsilon$ from $\lambda$.) See Figure 5 .

A key difference from the point case is that the edges of the geodesic ridge tree are not necessarily straight-line segments. Every edge of the ridge tree is the locus of points that are equidistant from two source images. Thus, when the two sources to either side of an edge are a point image and a segment image respectively, a parabolic ridge tree edge will result. The ridge tree edges between pairs of point images or pairs of segment images will still be straight. 


\section{New Proof for the Point Star Unfolding}

In this section we give a new proof of non-overlap for the point star unfolding that is much simpler than the original proof of Aronov and O'Rourke [2].

The most important idea of our proof is to partition the star unfolding into pairs of congruent triangles. Each vertex of the ridge tree has three or more shortest paths to $x$ on the surface of $P$. Add all these shortest paths as line segments in the star unfolding. Notice that each edge of the ridge tree will now have a triangle to each side. Because all shortest paths from a ridge tree vertex to the nearest source image have the same length, the two triangles to either side of a ridge tree edge have corresponding sides of equal length, and therefore are congruent. We call each such pair of triangles a kite. The kite associated with ridge tree edge $e$ is denoted kite $(e)$. The two images of $x$ to each side of $e$ are called the apices of the kite. Observe that the kites form a partition of $S_{x}$. See Figure 2(b).

We define the source angle of $e, \alpha(e)$, to be the interior angle at either apex of kite $(e)$. See Figure 2(b). We extend this definition to paths: For a path $\sigma$ of ridge tree edges, where $\sigma=e_{0}, \ldots, e_{t}$, the source angle of $\sigma$ is $\alpha(\sigma)=\sum_{i=0}^{t} \alpha\left(e_{i}\right)$. Observe that $\alpha(\sigma) \leq \pi$ because $2 \alpha(\sigma)$ measures the total source angle at both apices of every kite on the path, so this is bounded by the total surface angle at the source point $x$, which is bounded by $2 \pi$.

- Theorem 4 (Theorem 9.1 in [2]). The star unfolding $S_{x}$ does not overlap.

Proof. We will show that no two kites overlap. Consider two kites, and the path in the ridge tree between them. Let $e_{1}, \ldots, e_{t}$ be the edges of the path in the ridge tree, and let $k_{i}=k i t e\left(e_{i}\right)$. We will show that $k_{1}$ and $k_{t}$ have disjoint interiors. We will define a sequence of regions $W_{1}, \ldots, W_{t-1}$, called $\mathrm{W}$-wedges, so that $W_{i}$ includes $k_{1}, \ldots, k_{i}$ and excludes $k_{i+1}$. Then $W_{t-1}$ includes $k_{1}$ and excludes $k_{t}$, which will complete the proof.

The boundary of the W-wedge $W_{i}$ is shaped like a 'W' and defined as follows. The center point of $W_{i}$ is the point $p_{i}$, where $e_{i}$ and $e_{i+1}$ intersect; the inner legs are the edges of $k_{i}$ that are incident to $p_{i}$; and the outer legs form an angle with the inner legs (on the side of $k_{i}$ ) of $\frac{\pi}{2}+\alpha_{i}$, where $\alpha_{i}=\sum_{j=1}^{i} \alpha\left(e_{j}\right)$. The outer legs extend either to their point of intersection, or as infinite rays if they do not intersect. This boundary divides the plane into two regions and we define $W_{i}$ to be the region containing $k_{i}$ in a neighbourhood of $p_{i}$. See Figure 3 . Note that $\alpha_{i}$ is in the range $[0, \pi]$ as observed above.

We will prove by induction that $k_{i}$ is outside $W_{i-1}$ and that $W_{i}$ contains $k_{i} \cup W_{i-1}$. At each step, including the base case, we will need the following:

- Lemma 5. Let $p$ be an endpoint of ridge tree edge e, and let $W$ be a $W$-wedge centered at $p$ such that the inner legs of $W$ are the edges of kite(e), and the outer legs are rotated out (on the side of kite(e)) by an angle in the range $\left[\frac{\pi}{2}+\alpha(e), \frac{3 \pi}{2}\right]$. Then kite $(e) \subseteq W$.

Proof. Consider the two circular sectors centered at the endpoints $p$ and $q$ of $e$ and bounded by the two incident kite edges as radii (see for example the circular sector marked $a$ centered at $q=p_{0}$ on kite $k_{1}$ in Figure 3). At each apex of kite $(e)$ the two angles between the outer leg of $W$ and the two sides of kite $(e)$ are at least $\frac{\pi}{2}$. Thus the circular sector at $q$ is inside $W$, and the circular sector at $p$ is outside $W$. This implies that kite $(e) \subseteq W$.

We are now ready to prove by induction that $k_{i}$ is outside $W_{i-1}$ and that $W_{i}$ contains $k_{i} \cup W_{i-1}$. For the base case $i=1$, there is no $W_{0}$, and $W_{1}$ contains $k_{1}$ by the lemma above. Suppose by induction that $W_{i-1}$ contains $k_{i-1} \cup W_{i-2}$. We will show how to transform $W_{i-1}$ into $W_{i}$ in a way that makes it clear that $k_{i}$ is outside $W_{i-1}$ and $W_{i}$ contains $k_{i} \cup W_{i-1}$. 


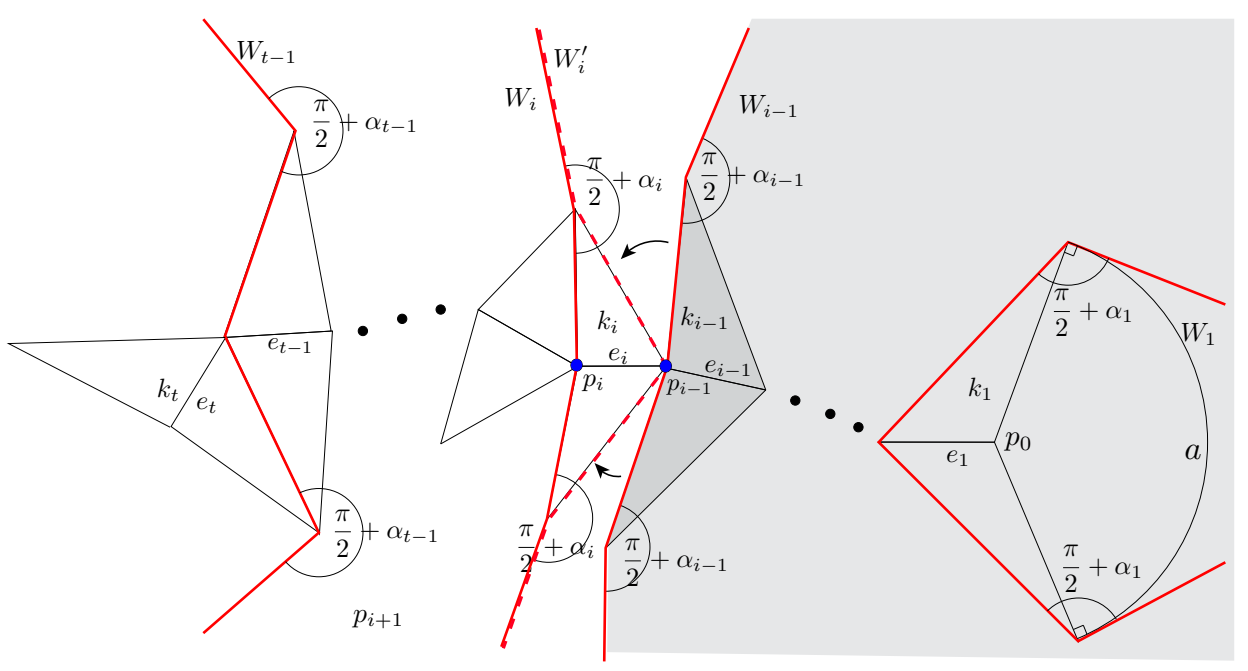

Figure 3 Kite $k_{i-1}$ (shaded) and the corresponding W-wedge $W_{i-1}$ (lightly shaded). $W_{1}$ contains kite $k_{1}$ because it contains the circular sector $a$. To prove by induction that $W_{i}$ contains all previous kites, we expand $W_{i-1}$, first rotating the legs about $p_{i-1}$ to the $\mathrm{W}$-wedge $W_{i}^{\prime}$ (dashed line), and then moving the center point to $p_{i}$ to obtain $W_{i}$. Note that although the figure shows kites $k_{i-1}$ and $k_{i}$ sharing only a vertex, in non-degenerate situations ridge tree vertices have degree 3 , and two consecutive kites will share an edge as well.

Note that the unfolding does not overlap in the neighbourhood of point $p_{i}-$ this is true whether $p_{i}$ is a point with $2 \pi$ surface area, or a vertex, which will be incident to a single cut. Thus the kites $k_{i}$ and $k_{i-1}$, which are both incident to $p_{i}$, do not overlap. Rotate the two inner legs of $W_{i-1}$ about point $p_{i-1}$, away from $k_{i-1}$ to the edges of $k_{i}$. Keep the angle between inner and outer legs fixed throughout the rotation. Observe that all the kite edges incident to $p_{i-1}$ have the same length, so we really perform a rigid transformation on each half of the W. Call the resulting W-wedge $W_{i}^{\prime}$ (shown as a dashed poly-line in Figure 3 ). Notice that $W_{i}^{\prime}$ contains $W_{i-1}$, because the angle $\alpha_{i-1}+\frac{\pi}{2}$ is in the range $\left[\frac{\pi}{2}, \frac{3 \pi}{2}\right]$ so the outer legs remain outside the rotation sector of the inner legs. (Here it is crucial that we added the extra $\frac{\pi}{2}$ to the initial angle.) That $k_{i}$ is outside $W_{i}^{\prime}$ follows from applying Lemma 5 to the outside of $W_{i}^{\prime}$, noting that $\alpha_{i-1}+\alpha\left(e_{i}\right) \leq \pi$ so the angle $\alpha_{i-1}+\frac{\pi}{2}$ is actually in the range $\left[\frac{\pi}{2}, \frac{3 \pi}{2}-\alpha\left(e_{i}\right)\right]$ and therefore the complementary angle is in the range $\left[\alpha\left(e_{i}\right)+\frac{\pi}{2}, \frac{3 \pi}{2}\right]$.

The second step of the transformation is to move the center point of $W_{i}^{\prime}$ from $p_{i-1}$ to $p_{i}$, while keeping the outer legs fixed. The $\mathrm{W}$-wedge increases until it contains $k_{i}$. The angle between inner and outer legs increases by $\alpha\left(e_{i}\right)$, to $\alpha_{i}$. Thus the result is precisely $W_{i}$, and therefore $W_{i}$ contains $k_{i} \cup W_{i-1}$.

Our proof, like Aronov and O'Rourke's, shows a stronger result that certain regions outside the star unfolding are empty. Aronov and O'Rourke [2] showed that at any vertex $v_{i}$ adjacent to source images $x_{i}$ and $x_{i+1}$ in the unfolding, no part of the unfolding enters the circular sector centered at $v_{i}$ exterior to the unfolding near $v_{i}$ and bounded by the radii $v_{i} x_{i}$ and $v_{i} x_{i+1}$. See Figure 4 . Our proof shows that larger regions are empty:

Lemma 6. Let $v_{i}$ be a vertex adjacent to source images $x_{i}$ and $x_{i+1}$ in a star unfolding $S_{x}$, and let $W$ be the region bounded by $v_{i} x_{i}, v_{i} x_{i+1}$, and the rays extending from $x_{i}$ and $x_{i+1}$ at right angles from these segments on the exterior side of $v_{i}$. Then no part of the unfolding intersects the interior of $W$. 


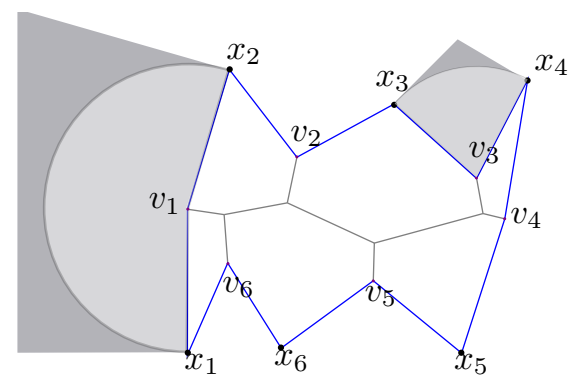

Figure 4 Old sectors of emptiness (light grey) established by Aronov and O'Rourke and new empty regions (light grey+dark grey) established by Lemma 6 .

Proof outline. In non-degenerate situations, $v_{i}$ is a leaf of the ridge tree, incident to ridge tree edge $e$, say. We apply the argument in the proof above to the ridge tree path from $e$ to any other edge. The initial $\mathrm{W}$-wedge always contains $W$, so no other kite enters $W$. More generally, $v_{i}$ may be an internal vertex of the ridge tree and we repeat the argument for every path in the ridge tree starting at $v_{i}$, taking the intersection of the initial wedges in the path argument to show that $W$ is empty.

\section{Geodesic Star Unfolding}

In this section we will consider the star unfolding from a geodesic curve $\lambda$. By generalizing the proof for the point case, we will establish some situations in which the geodesic star unfolding does not overlap. See Figure 5.

As for the point-source case, we will partition the unfolding by adding, for every ridge tree vertex $p$, the line segments that correspond to shortest paths from $p$ to the curve. We include as a vertex of the ridge tree any point of the ridge tree that has a shortest path to an endpoint of $\lambda$ appearing in a segment image. The added line segments partition the unfolding into regions called wings. See Figure 5(b). The two wings on either side of a ridge tree edge form a wing-pair. A wing with an endpoint source image is a point wing and a wing with a segment source image is a segment wing. A wing-pair may involve two point wings (these are the kites of the previous section), or two segments wings, or one of each, in which case we call it a hybrid wing-pair. The ridge tree edge of a hybrid wing-pair is a parabolic segment; all others are straight-line segments.

The source angle of a point wing is the angle at its source image point; the source angle of a segment wing is 0 . The source angle of a wing-pair is the sum of the source angles of the two wings. If $e$ is an edge of the ridge tree, and $A$ designates one side of this edge, then $\alpha^{A}(e)$ denotes the source angle of the wing on that side. If $\sigma$ is a path in the ridge tree, with its two sides (arbitrarily) labelled $A$ and $B$, then the source angle of $\sigma$ on side $A$ is $\alpha^{A}(\sigma)=\sum_{i=0}^{t} \alpha^{A}\left(e_{i}\right)$ (and similarly for $B$ ). The path $\sigma$ is balanced if $\alpha^{A}(\sigma) \leq \pi$ and $\alpha^{B}(\sigma) \leq \pi$. We say that the ridge tree [or the geodesic curve] is balanced if every path in the ridge tree is balanced. There are examples where the ridge tree is not balanced, and in fact it is possible to have all $2 \pi$ of source angle to one side of a ridge tree path, see [10].

Our main result in this section is that wing-pairs along a balanced path do not overlap.

- Lemma 7. Let $\mathcal{P}$ be a convex polyhedron with a geodesic curve $\lambda$ on its surface. Suppose that $u$ and $v$ are distinct edges of the ridge tree such that the path in the ridge tree from $u$ to $v$ is balanced. Then the wing-pairs of $u$ and $v$ do not overlap. 


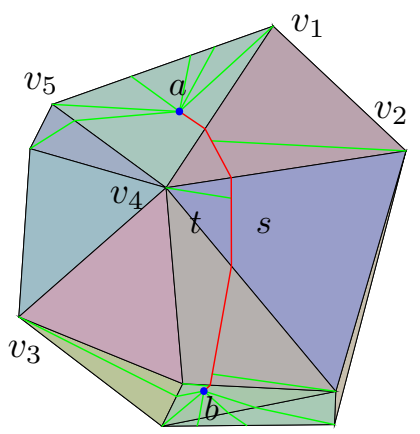

(a)

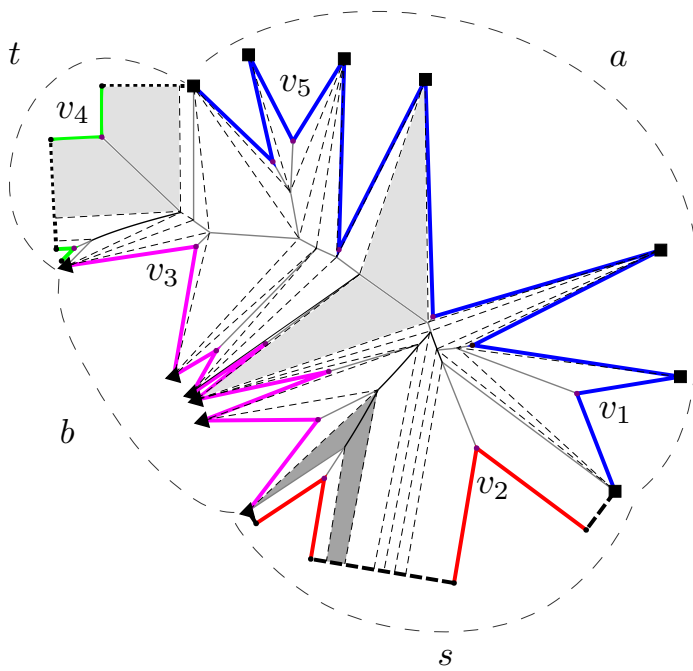

(b)

Figure 5 The star unfolding from a geodesic curve. (a) The polyhedron, the geodesic curve with endpoints $a$ and $b$ and sides $s$ and $t$, and shortest paths from vertices to the curve. (b) The corresponding star unfolding with the source images, $a, b, s$ or $t$, indicated. Images of $a[b]$ are drawn as squares [triangles]; segment images of $s[t]$ are drawn as heavy dashed [dotted] lines. Shortest path cuts are coloured according to their destination type. Wings are indicated by dashed lines. Three wing-pairs are shaded; the darkly shaded one is a hybrid wing-pair with a parabolic ridge tree edge. Note that the unfolding shows the inside surface of the polyhedron.

Before proceeding with the proof, we note the consequence that the star unfolding from a balanced geodesic curve does not overlap:

- Corollary 8. If $\mathcal{P}$ is a convex polyhedron with a balanced geodesic curve $\lambda$ then the geodesic star unfolding from $\lambda$ does not overlap.

Proof of Lemma. We follow the same plan as in the proof of Theorem 4, that is, we will prove that no two wing-pairs in the unfolding overlap, by examining $\mathrm{W}$-wedges along the ridge tree path between the two wing-pairs. A quick summary is that there are only two differences in the argument: (1) as we move from $\mathrm{W}$-wedge $W_{i-1}$ to $W_{i}$ we may increase the angle between inner and outer legs differently on its two sides; (2) when the W-wedge moves past a segment wing, the angle between inner and outer legs does not change, and the inner+outer pair translates. See Figure 6 . We now give the details.

Let $\sigma$ be the path from $u$ to $v$ in the ridge tree, with edges $u=e_{1}, \ldots, e_{t}=v$. Let $w_{i}$ be the wing-pair of edge $e_{i}$. Label the two sides of $\sigma$ by $A$ and $B$. Let $\alpha_{i}^{A}=\sum_{j=1}^{i} \alpha^{A}\left(e_{j}\right)$ and let $\alpha_{i}^{B}=\sum_{j=1}^{i} \alpha^{B}\left(e_{j}\right)$. Note that $\alpha_{i}^{A}$ and $\alpha_{i}^{B}$ are in the range $[0, \pi]$ by assumption.

We will show that $w_{1}$ and $w_{t}$ have disjoint interiors by defining $\mathrm{W}$-wedges $W_{i}$ so that $W_{i}$ includes $w_{1}, \ldots, w_{i}$ and excludes $w_{i+1}$. Then $W_{t-1}$ includes $w_{1}$ and excludes $w_{t}$, which will complete the proof. Define $W_{i}$, for $i=1, \ldots, t$, to be the $\mathrm{W}$-wedge with center point at $p_{i}$ where $e_{i}$ and $e_{i+1}$ intersect, with inner legs along the two incident edges of $w_{i}$, and outer legs rotated out (on the side of $w_{i}$ ) by $\alpha_{i}^{A}+\frac{\pi}{2}$ on side $A$, and by $\alpha_{i}^{B}+\frac{\pi}{2}$ on side $B$. The outer legs extend either to their point of intersection, or as infinite rays if they do not intersect. This boundary divides the plane into two regions and we define $W_{i}$ to be the region containing $w_{i}$. See Figure 6 . 


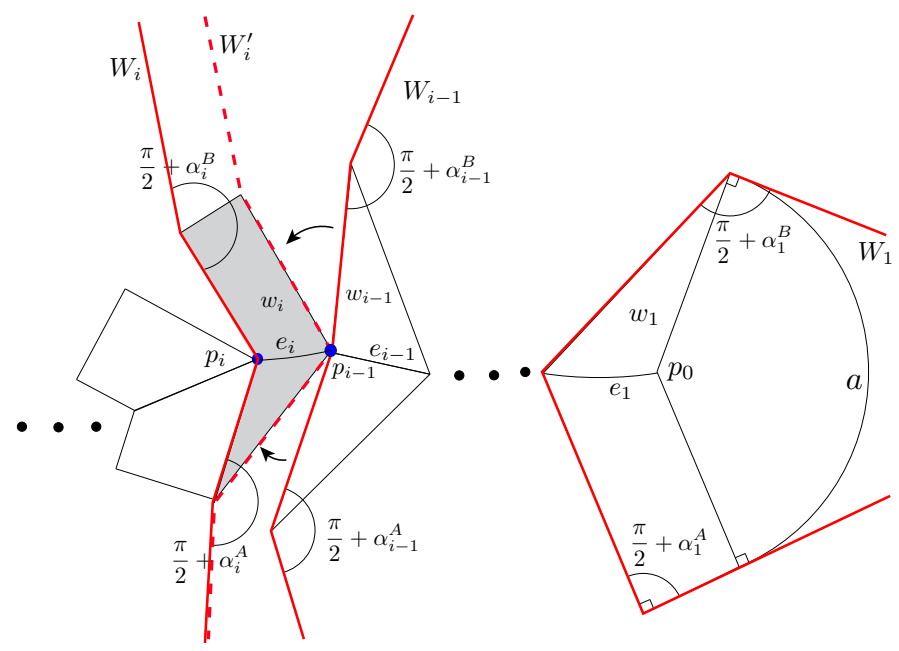

Figure 6 Wing-pair $w_{i}$ (shaded) is a hybrid wing-pair. $W_{1}$ contains $w_{1}$ because it contains the circular sector $a$. To prove by induction that $W_{i}$ contains all previous wing-pairs, we expand $W_{i-1}$ to $W_{i}$, first rotating the legs about $p_{i-1}$ to the $\mathrm{W}$-wedge $W_{i}^{\prime}$ (dashed line), and then expanding to include $w_{i}$. Note that in this example $\alpha_{i-1}^{B}=\alpha_{i}^{B}$ since the wing to that side is a segment-wing.

We will prove by induction that $w_{i}$ is outside $W_{i-1}$ and that $W_{i}$ contains $w_{i} \cup W_{i-1}$. At each step, including the base case, we will need the following:

- Lemma 9. Let $p$ be an endpoint of ridge tree edge e, and let $W$ be a $W$-wedge centered at $p$ such that the inner legs of $W$ are the edges of the incident wings of e, and the outer leg on the $A$ side is rotated out by an angle in the range $\left[\frac{\pi}{2}+\alpha^{A}(e), \frac{3 \pi}{2}\right]$ and the outer leg on the $B$ side is rotated out by an angle in the range $\left[\frac{\pi}{2}+\alpha^{B}(e), \frac{3 \pi}{2}\right]$. Then the wing-pair of $e$ is contained in $W$.

Proof. Consider the two circular sectors centered at the endpoints $p$ and $q$ of $e$ and bounded by the two incident wing edges as radii (see for example the circular sector marked $a$ centered at $q=p_{0}$ on wing-pair $w_{1}$ in Figure 6 ). On both the $A$ side and the $B$ side, the angles between the outer leg of $W$ and the sides of the wing of $e$ are at least $\frac{\pi}{2}$. Thus the circular sector at $q$ is inside $W$, and the circular sector at $p$ is outside $W$. This implies that the wing-pair of $e$ is contained in $W$.

We are now ready to prove by induction that $w_{i}$ is outside $W_{i-1}$ and that $W_{i}$ contains $w_{i} \cup W_{i-1}$. For the base case $i=1$, there is no $W_{0}$, and $W_{1}$ contains $w_{1}$ by the lemma above. Suppose by induction that $W_{i-1}$ contains $w_{i-1} \cup W_{i-2}$. We will show how to transform $W_{i-1}$ into $W_{i}$ in a way that makes it clear that $w_{i}$ is outside $W_{i-1}$ and $W_{i}$ contains $w_{i} \cup W_{i-1}$.

Since there is at most $2 \pi$ surface angle at any point on the surface, the unfolding does not overlap in the neighbourhood of any point. Thus $w_{i-1}$ and $w_{i}$ are disjoint. Rotate the two inner legs of $W_{i-1}$ about point $p_{i-1}$, away from $w_{i-1}$ to the edges of $w_{i}$. Keep the angle between inner and outer legs fixed throughout the rotation. Observe that all the wing edges incident to $p_{i-1}$ have the same length, so we really perform a rigid transformation on each half of the W. Call the resulting W-wedge $W_{i}^{\prime}$ (shown as a dashed poly-line in Figure 6). Notice that $W_{i}^{\prime}$ contains $W_{i-1}$, because the angles $\alpha_{i-1}^{A}+\frac{\pi}{2}$, and $\alpha_{i-1}^{B}+\frac{\pi}{2}$ are in the range $\left[\frac{\pi}{2}, \frac{3 \pi}{2}\right]$ so the outer legs remain outside the rotation sector of the inner legs. We prove that $w_{i}$ is outside $W_{i}^{\prime}$ by applying Lemma 9 to the outside of $W_{i}^{\prime}$. To show that the angle on 


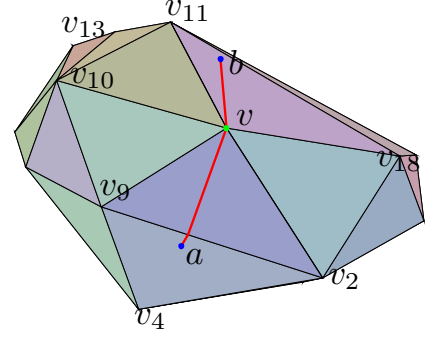

(a)

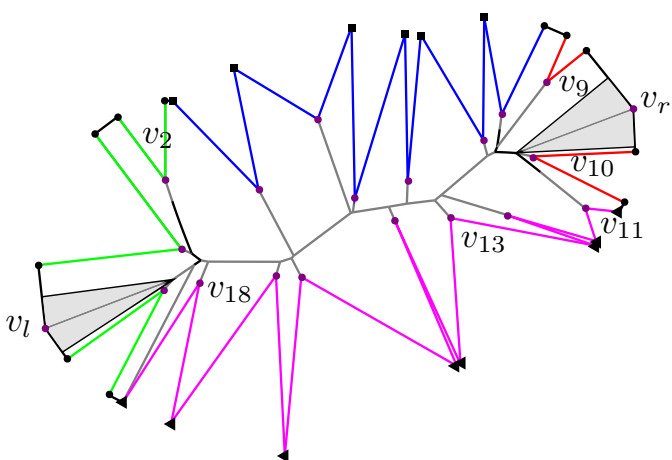

(b)

Figure 7 The star unfolding from a quasi-geodesic curve. (a) The polyhedron and the quasigeodesic curve passing through vertex $v$. (b) The corresponding star unfolding with the two images of $v$ and their wing-pairs indicated.

the $A$ side is in the required range, observe that $\alpha_{i-1}^{A}+\alpha^{A}\left(e_{i}\right) \leq \pi$ so the angle $\alpha_{i-1}^{A}+\frac{\pi}{2}$ is actually in the range $\left[\frac{\pi}{2}, \frac{3 \pi}{2}-\alpha^{A}\left(e_{i}\right)\right]$ and therefore the complementary angle is in the range $\left[\alpha^{A}\left(e_{i}\right)+\frac{\pi}{2}, \frac{3 \pi}{2}\right]$. A similar argument applies on the $B$ side.

The second step of the transformation is to move $W_{i}^{\prime}$ past $w_{i}$ to $W_{i}$. We do this separately on the $A$ side and the $B$ side. To go past a point wing, we move the inner leg from $p_{i-1}$ to $p_{i}$, while keeping the outer leg fixed. To go past a segment wing, we translate the inner+outer legs so that their common point moves along the segment image; since the segment image is perpendicular to the inner leg, each leg is parallel to its initial version. The resulting $\mathrm{W}$-wedge contains $w_{i}$. On the $A$ side, the angle between inner and outer legs increases by $\alpha^{A}\left(e_{i}\right)$, to $\alpha_{i}^{A}$, and similarly on the $B$ side. Thus the result is precisely $W_{i}$, and therefore $W_{i}$ contains $w_{i} \cup W_{i-1}$.

\subsection{Extension to Quasigeodesic Curves}

We now show that our geodesic star unfolding result (Lemma 7) carries over to quasigeodesic curves. Recall that a quasigeodesic curve on the surface of a polyhedron $\mathcal{P}$ is a curve such that at each interior point along the curve the surface angle to each side is $\leq \pi$. (The angle can only be $<\pi$ at a vertex of $\mathcal{P}$.)

Consider a quasigeodesic curve $\lambda$ on the surface of $\mathcal{P}$. We define the quasigeodesic star unfolding in the same way as the geodesic star unfolding, specifically, we cut the curve and a shortest path from every vertex to the curve. See Figure 7.

To argue about the ridge tree of $\lambda$, we consider the closed curve consisting of the points at some small fixed distance $\epsilon$ from $\lambda$. This curve is composed of circular arcs and geodesic segments joined at angles $\leq \pi$ (on the side opposite $\lambda$ ). Thus by [4, Lemma 4], its ridge tree is a tree. The ridge tree of $\lambda$ itself (when $\epsilon=0$ ) has the peculiarity that it has a cycle if $\lambda$ has an interior point with surface angle $<\pi$ on both sides of the curve (e.g., vertex $v$ in Figure 7). However, we split any such vertex into two copies, corresponding to the $s$ and $t$ sides of the curve, which breaks the cycle in the unfolded ridge tree.

Suppose $p$ is an interior point of the quasigeodesic curve $\lambda$ where the surface angle to one side, say the $s$ side, is $\beta$, where $\beta<\pi$. Necessarily, $p$ is a vertex of the polyhedron, otherwise the surface angle on the other side of the curve would be greater than $\pi$. We do not introduce a cut for this vertex in the unfolding, since it already lies on $\lambda$. 
Using Lemma 3, we claim that no shortest path cut from any vertex $v$ will report to point $p$ on side $s$, since one of the two surface angles formed would be $<\frac{\pi}{2}$. Thus the quasigeodesic star unfolding from $\lambda$ will have a vertex image with an angle $\beta$ corresponding to the $s$ side of $p$. Let $p_{s}$ denote this vertex image in the unfolding.

Observe that $p_{s}$ is a leaf of the unfolded ridge tree and that the incident ridge tree edge $e$ is a straight segment forming angles $\frac{\beta}{2}$ with the segment images to either side of $p_{s}$. We can consider $e$ to have two segment wings, albeit degenerate ones, with one side (between $p_{s}$ and $\lambda$ ) of length 0 . We call this a quasi-wing-pair (see the shaded examples in Figure 7).

With these observations in hand, we can extend the result of the previous section to quasigeodesics. The main idea is to show that quasi-wing pairs can only occur as the first or last wing-pair along a path of the unfolded ridge tree, and thus that the induction proof of the previous section carries over.

- Lemma 10. Let $\mathcal{P}$ be a convex polyhedron with a quasigeodesic curve $\lambda$ on its surface. Suppose that $u$ and $v$ are distinct edges of the ridge tree such that the path in the unfolded ridge tree from $u$ to $v$ is balanced. Then the (quasi-)wing-pairs of $u$ and $v$ do not overlap.

\subsection{Quasigeodesic Loops}

In this section, we prove non-overlap of the star unfolding from geodesic (and quasigeodesic) loops. When the two endpoints $a$ and $b$ of a geodesic or quasigeodesic curve $\lambda$ coincide at point $o$, we call this a (quasi)geodesic loop with loop point o. A (quasi)geodesic loop cuts the surface of the polyhedron into two pieces. One piece must have a surface angle at $o$ that is $\leq \pi$, and we call this the inside of the loop and identify it with the $s$ side of the curve. The other piece is called the outside and will be identified with the $t$ side of the curve.

Itoh, O'Rourke, and Vîlcu [9] proved that for any quasigeodesic loop: (1) the inside unfolds without overlap; (2) the outside unfolds without overlap; and (3) the two unfolded pieces can be reattached (without overlap) along a common segment of the cut curve. Their proof of (2) relies on a Lemma [9, Lemma 7] about the star unfolding from a point, which they say will be proved in a companion paper, but unfortunately, they discovered ${ }^{1}$ that the Lemma is false. The Lemma claims that for any star unfolding from a point $x$ and for any polyhedron vertex $v$, the exterior angle at $v$ in the unfolding yields an empty wedge. More precisely, if the exterior angle at $v$ is (counterclockwise) $x_{i}, v, x_{i+1}$ then the claim is that the counterclockwise wedge formed by the rays $v x_{i}$ and $v x_{i+1}$ does not contain any part of the unfolding. An example where this fails is shown in Figure 8.

In this section we examine the star unfolding from a quasigeodesic curve where the two endpoints, $a$ and $b$, of the curve are arbitrarily close together. In the limit when $a=b$ the unfolding consists of two pieces joined at the point $a=b$ with the angular bisectors at the point $a=b$ aligned in the unfolding. We call this the conjoined star unfolding from a quasigeodesic loop. See Figure 9. Our main result of this section is that the conjoined star unfolding from a quasigeodesic loop does not overlap. This implies that the outside piece unfolds without overlap, which repairs the missing step of Itoh, O'Rourke and Vîlcu's result.

- Theorem 11. The conjoined star unfolding from a quasigeodesic loop does not overlap.

We prove Theorem 11 by showing that every path through the ridge tree for a geodesic loop is balanced. Then by Lemma 7, the unfolding does not overlap. Furthermore, by Lemma 10, the result extends to quasigeodesics.

1 Private communication from J. O'Rourke 


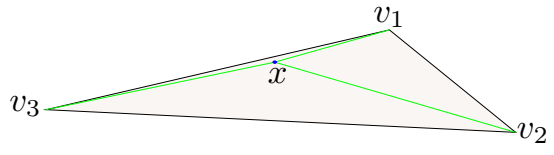

(a)

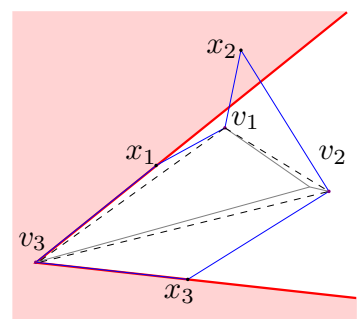

(b)

Figure 8 A counterexample to [9, Lemma 7]. (a) A doubly covered triangle with source point $x$. (b) The star unfolding from $x$ showing a wedge formed by the exterior angle $x_{3}, v_{3}, x_{1}$ that intersects the unfolding. Dashed edges show the back face; grey edges show the ridge tree.

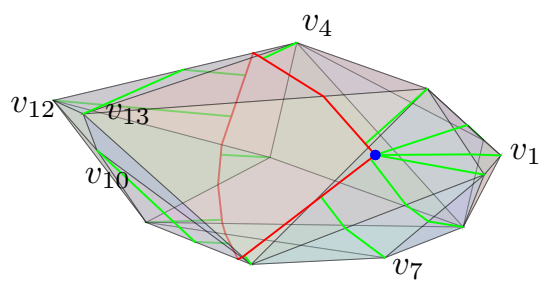

(a)

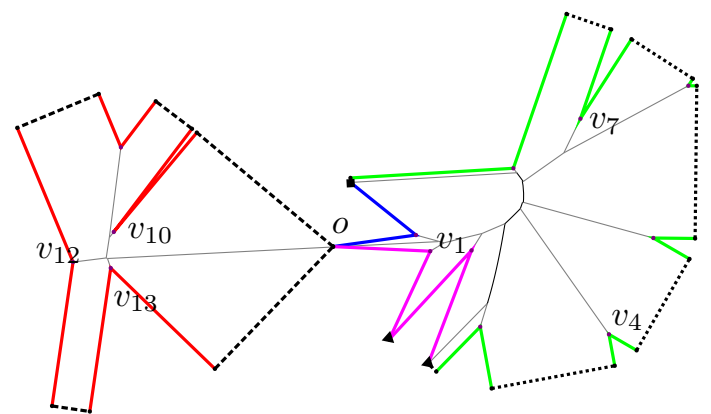

(b)

Figure 9 Conjoined star unfolding from a geodesic loop. (a) Geodesic loop on the surface of a polyhedron (partially transparent to view the full loop). (b) Unfolding.

Lemma 12. Every path through the unfolded ridge tree of the conjoined star unfolding from a quasigeodesic loop is balanced.

Proof. Recall our assumption that the $s$-side of the curve is inside the loop. By Lemma 3, no vertex inside the loop can report to the loop point $o$, since the surface angle to the interior of the loop is $<\pi$ (or if it is equal to $\pi$, we can assume the vertex reports to $b$ instead).

Consider the segment of the ridge tree (call it $u$ ) that lies between $a$ and $b$ on the inside of the loop and touches loop point $o$. Observe what happens in the limit as $a$ and $b$ approach point $o$. Consider the kite-shaped region of the surface delimited by $a, o, b$, and $p$, where $p$ is the intersection between the rays perpendicular to the geodesic at $a$ and $b$ respectively (see Figure 10). Call this region $R$. When $a$ and $b$ are close enough to $o$ there are no vertices or other ridge tree edges inside the region $R$, and therefore some sub-segment of $u$ will extend from $p$ to $o$ (i.e., perpendicularly bisect $\overline{a b}$ ). Therefore the source angle of each wing of $u$ is at least $\frac{\pi}{2}$, and the source angle of $u$ 's wing-pair is at least $\pi$. Furthermore, this is the only edge of the ridge tree on the inside of the loop that has point-wings reporting to $a$ or $b$.

Consider any path $\sigma$ through the unfolded ridge tree. Let $A$ and $B$ be the sides of $\sigma$. We must show that $\alpha^{A}(\sigma) \leq \pi$ and $\alpha^{B}(\sigma) \leq \pi$. There are three possible cases for the path:

1. The path $\sigma$ does not include $u$, and remains entirely on the inside of the loop. Because $u$ is the only ridge tree edge inside the loop whose wings have non-zero source angle, therefore $\alpha^{A}(\sigma)=\alpha^{B}(\sigma)=0$. 


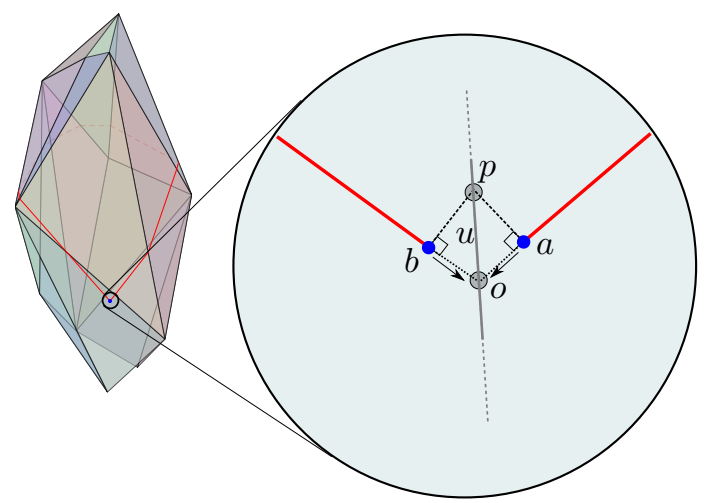

Figure 10 Zoomed-in view of the surface as $a$ and $b$ approach $o$. Assuming no vertices are inside the region aobp, there is at least $\frac{\pi}{2}$ source angle to either side of ridge tree edge $u$.

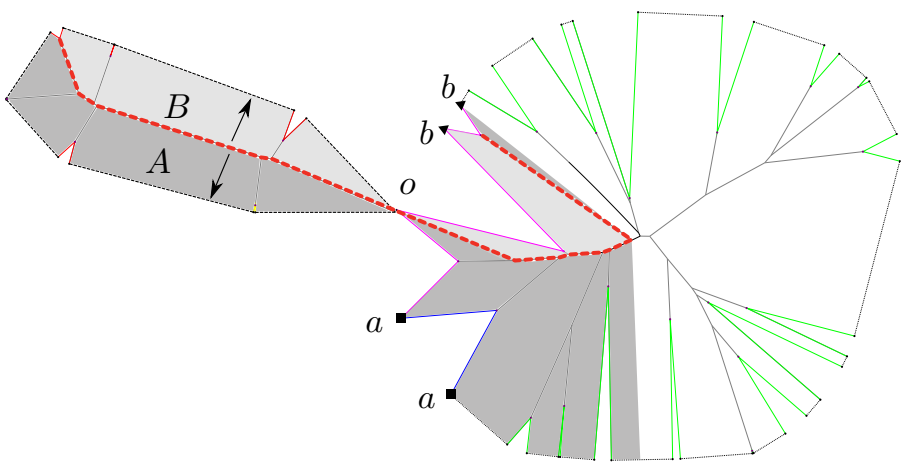

Figure 11 An illustration of the third case in the proof of Lemma 12, where the $A$ side of the path $\sigma$ (in heavy dashed red) has point wings reporting to $a$ and to $b$.

2. The path $\sigma$ does not include $u$, and remains entirely on the outside of the loop. As noted above, the source angle of $u$ 's wing-pair is at least $\pi$. Therefore, the remaining source angle of all wings along every other possible path (i.e., not including $u$ ) must be $\leq \pi$. Thus $\alpha^{A}(\sigma) \leq \pi$ and $\alpha^{B}(\sigma) \leq \pi$.

3. The path $\sigma$ includes $u$. We must show that $\alpha^{A}(\sigma) \leq \pi$ and $\alpha^{B}(\sigma) \leq \pi$. Any edge of $\sigma$ (apart from $u$ ) that lies inside the geodesic loop only has segment wings to either side and these contribute 0 to the source angle of the path. Thus it suffices to look at the portion of $\sigma$ starting with $u$ and then following ridge tree edges that lie outside the geodesic loop. Call this subpath $\sigma^{\prime}$. Ridge tree edge $u$ has a point wing to either side, one reporting to $a$ and one reporting to $b$. Suppose that the $A$ side of the path has the point wing reporting to $a$. Suppose that $\alpha^{A}\left(\sigma^{\prime}\right) \geq \alpha^{B}\left(\sigma^{\prime}\right)$. If side $A$ only has point wings that report to $a$, then its source angle is at most $\pi$. So suppose that side $A$ has a point wing that reports to $b$. See Figure 11. As we walk around the path $\sigma^{\prime}$, in counterclockwise order starting with $u$ on the $A$ side, the wings report in order to $a$, then $t$, then $b$. Thus all the wings on the $B$ side must be point wings that report to $b$, which implies that the sum of their source angles is at most $\pi$, i.e., $\alpha^{B}\left(\sigma^{\prime}\right) \leq \pi$. Since every wing on the $B$ side is a point-wing reporting to $b$, every point wing on the $A$ side must be paired with a point wing on the $B$ side, and each such pair has equal source angles. Therefore $\alpha^{A}\left(\sigma^{\prime}\right) \leq \alpha^{B}\left(\sigma^{\prime}\right)$, and we just showed this is $\leq \pi$. 


\subsection{The Geodesic Star Unfolding as Two Non-overlapping Pieces}

Although we have not proved that the geodesic star unfolding never overlaps, in this section we show that it can always be cut into two pieces each of which is non-overlapping. The extra cuts consist of two shortest paths from a point on the ridge tree to the geodesic curve.

- Lemma 13. Let $\mathcal{P}$ be a convex polyhedron and $\lambda$ be a geodesic curve on $\mathcal{P}$. Then there is a point $p$ of the ridge tree of $\lambda$ such that cutting two shortest paths on $\mathcal{P}$ from $p$ to the geodesic curve $\lambda$ separates the geodesic star unfolding $S_{\lambda}$ into two pieces each of which is non-overlapping.

Proof. We will split the ridge tree into two subtrees, either at an internal point of an edge or at a ridge vertex $v$, in which case we split the ridge tree edges incident to $v$ into two subsets, each consecutive in the cyclic order of edges around $v$. Call such subtrees proper. The geodesic star unfolding $S_{\lambda}$ can then be cut into two pieces as follows: if the two subtrees are joined at an internal point $p$ of a ridge tree edge, then we cut the two shortest paths from $p$ to the geodesic curve $\lambda$; and if the two subtrees are joined at vertex $v$ then we cut the two shortest paths from $v$ to $\lambda$ that separate the incident ridge tree edges as specified. This ensures that if two wing-pairs are in the same piece of the unfolding, then the ridge tree path between them lies in the same subtree. So long as each subtree is balanced, Lemma 7 ensures that no two wing-pairs from the same piece overlap, i.e., that each piece is non-overlapping.

In the remainder of the proof we show how to partition the ridge tree into two proper balanced subtrees. Each edge $e$ of the ridge tree has an associated source angle of its wing pair, $\sigma(e)$, and the sum of these weights over the whole ridge tree is $2 \pi$. We remark that a weaker form of the lemma with three non-overlapping pieces can be obtained from the result that any edge-weighted tree can be separated at a single vertex into three subtrees of weight at most one half the total weight. To prove the lemma we will argue about the source angles on each side of each ridge tree edge.

Among all proper subtrees of the ridge tree, let $T$ be a maximal subtree that is balanced. Let $\bar{T}$ be the complement. We claim that $\bar{T}$ is balanced.

If the source angle of $T$ is at least $\pi$, then $\bar{T}$ has source angle $\leq \pi$ so it must be balanced as well. Otherwise the source angle of $T$ is $<\pi$. Note that $T$ cannot be rooted at an interior point of an edge otherwise we could move the point further along the edge to increase the source angle of $T$ a small amount without exceeding $\pi$. Therefore $T$ must be rooted at a vertex $v$ of the ridge tree. Among the edges incident to $v$ in clockwise order, let $e$ and $f$ be the first and last edges outside $T$. Note that $e \neq f$ (i.e., there is more than one edge incident to $v$ in $\bar{T}$ ) otherwise we could move the root of $T$ along $e$ to increase the source angle by a small amount. Adding $e$ and its subtree to $T$ gives an unbalanced subtree, so there must be an unbalanced path $\mu_{e}$ that contains $e$ and a subpath in $T$. Similarly, there must be an unbalanced path $\mu_{f}$ that contains $f$ and a subpath in $T$.

Note that any two unbalanced path-sides must have a wing in common, otherwise we would have two disjoint sets of wings each with source angle greater than $\pi$. Thus the unbalanced sides of $\mu_{e}$ and $\mu_{f}$ must both lie on the clockwise side of $e$ and $f$ or both on the counterclockwise side of $e$ and $f$ (relative to the cyclic ordering of edges at $v$ ). Suppose the former, without loss of generality.

Suppose that $\bar{T}$ has an unbalanced path $\mu$. The unbalanced side must share wings with the unbalanced side of $\mu_{e}$ and with the unbalanced side of $\mu_{f}$, and therefore must include the clockwise sides of both $e$ and of $f$, which is impossible. Therefore $\bar{T}$ is balanced, and we can separate the geodesic star unfolding $S_{\lambda}$ into two pieces each of which is non-overlapping. 


\section{Conclusions}

We have given a simple proof that the star unfolding from a point does not overlap, and extended it to some cases of the star unfolding from a geodesic curve. We leave open the main conjecture that the geodesic star unfolding does not overlap. All we can say about the general case is that the unfolding can be partitioned into two non-overlapping pieces.

The first author's thesis [10] contains further results on geodesics that have been "fully extended" until the endpoints $a$ and $b$ intersect the curve. When the endpoints reach opposite sides of the curve ("S-shaped") the unfolding does not overlap because it is balanced. When the endpoints reach the same side of the curve ("C-shaped") the unfolding need not be balanced, though some special cases can still be proved to avoid overlap.

The figures in this paper were generated with a custom program written using CGAL, OpenGL, and Cairo. For more information, see the first author's thesis [10].

Acknowledgements. We thank Timothy Chan for suggesting Lemma 13. We thank Joseph O'Rourke, Costin Vîlcu, and anonymous referees for helpful comments.

\section{References}

1 Pankaj K. Agarwal, Boris Aronov, and Catherine A. Schevon. Star unfolding of a polytope with applications. SIAM Journal on Computing, 26:1689-1713, 1997.

2 Boris Aronov and Joseph O'Rourke. Nonoverlap of the star unfolding. Discrete \& Computational Geometry, 8(3):219-250, 1992.

3 Erik D. Demaine, Martin L. Demaine, Anna Lubiw, Arlo Shallit, and Jonah Shallit. Zipper unfoldings of polyhedral complexes. In Proceedings of the 22nd Annual Canadian Conference on Computational Geometry (CCCG), pages 219-222, August 2010.

4 Erik D. Demaine and Anna Lubiw. A generalization of the source unfolding of convex polyhedra. In Revised Papers from the 14th Spanish Meeting on Computational Geometry (EGC 2011), volume 7579 of Lecture Notes in Computer Science, pages 185-199, Alcalá de Henares, Spain, June 27-30 2012.

5 Erik D. Demaine and Joseph O'Rourke. Geometric Folding Algorithms: Linkages, Origami, Polyhedra. Cambridge University Press, New York, NY, USA, 2007.

6 Albrecht Dürer. The Painter's Manual: A Manual of Measurement of Lines, Areas, and Solids by Means of Compass and Ruler Assembled by Albrecht Dürer for the Use of All Lovers of Art with Appropriate Illustrations Arranged to be Printed in the Year MDXXV. The literary remains of Albrecht Dürer. Abaris Books, 1977.

7 Kouki Ieiri, Jin-ichi Itoh, and Costin Vîlcu. Quasigeodesics and farthest points on convex surfaces. Advances in Geometry, 11(4):571-584, 2011.

8 Jin-Ichi Itoh, Joseph O'Rourke, and Costin Vîlcu. Source unfoldings of convex polyhedra with respect to certain closed polygonal curves. In Proceedings of the 25th European Workshop on Computational Geometry (EuroCG), pages 61-64, 2009.

9 Jin-ichi Itoh, Joseph O'Rourke, and Costin Vîlcu. Star unfolding convex polyhedra via quasigeodesic loops. Discrete \& Computational Geometry, 44(1):35-54, 2010.

10 Stephen Kiazyk. The star unfolding from a geodesic curve. Master's thesis, Cheriton School of Computer Science, University of Waterloo, 2014.

11 Joseph O'Rourke and Costin Vîlcu. Development of curves on polyhedra via conical existence. Computational Geometry, 47(2, Part A):149-163, 2014.

12 Micha Sharir and Amir Schorr. On shortest paths in polyhedral spaces. SIAM Journal on Computing, 15(1):193-215, 1986. 